\title{
Energy Efficient and Hybrid Bio-inspired Ant-Cuckoo Algorithm for Wireless Sensor Network
}

\author{
Viswanathan Chakravarthi', K.Gunasekaran ${ }^{2}$ Nijandan.S3 and S. Devikala ${ }^{4}$ \\ ${ }^{1}$ Chennai Institute of Technology, Chennai, India \\ ${ }^{2}$ Siddhartha Institute of Science and Technology, Puttur, AP, India, \\ ${ }^{3}$ GRT Institute of Engineering and Technology, Tiruthani, India \\ ${ }^{4}$ Varaprasad Reddy Institute of Technology, Guntur, AP, India
}

\section{ABSTRACT}

To develop the energy efficient protocol hybrid bio-inspired Ant-Cuckoo algorithm is proposed here to balance the energy consumption between the nodes evenly. The clusters are formed in the network using ACO algorithm. This is done based on the distance measurement among the nodes. Once the cluster is formed then the cluster head is selected upon the node energy value. Here flexible and rigid threshold is set for processing successive rounds of transmission with the same cluster head to avoid the frequent cluster reformation. Data routing using hauler nodes are selected with the assistance of cluster heads is done by using cuckoo search algorithm. Thereby efficient routes with reliable nodes are selected for data transmission. Simulation results are shown for proving efficiency for the proposed method.

KEY WORDS: ANT COLONY OPTIMIZATION; CUCKOO SEARCH; CLUSTER; ENERGY EFFICIENCY; WIRELESS SENSOR NETWORKS.

\section{INTRODUCTION}

WSN network is generally created with numerous numbers of nodes and mainly play with the sensor role. Significant importance is given to the energy efficiency since to improve the network lifetime. The recent techniques mainly focus on bio-inspired algorithms to improve the energy efficiency (Mochalov et al., 2015). The real time application gets wider since using bio-inspired algorithm (Bhatia et al., 2016) in WSN and this makes the network more secured as well (Izadi et al., 2015). WSN provides lot of services and is applicable for various fields by its nature, however faces many technical issues like energy balancing, load balancing, etc (Kumar et al., 2015).

Biosc Biotech Res Comm P-ISSN: 0974-6455 E-ISSN: 2321-4007
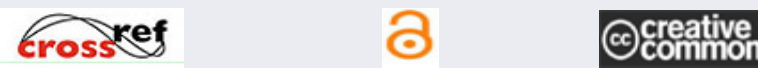

Identifiers and Pagination

Article Information

Year: 2021 Vol: 14 No (6) Special Issue

Pages: $335-338$

Received: $15^{\text {th }}$ April 2021

This is an open access article under Creative

Commons License Attribn 4.0 Intl (CC-BY).

DOI: $h t t p: / / d x$.doi.org/10.21786/bbrc/14.6.69
Bio-inspired evolutionary algorithms are meta-heuristic population-based optimization algorithms inspired by behavior of birds, animals, insect, genes etc. Bio- inspired algorithm such as Ant colony, Genetic Algorithm (Lv et al., 2017), Particle Swarm Optimization (PSO) (Yuan et al., 2017), Cuckoo Search optimization, Artificial Bee colony (ABC) (Rostami et al., 2014), Bat optimization, and many more evolutionary algorithms were getting large reputation in WSN as well as in other fields.

Related Works: Most of the energy efficiency techniques were proposed for the wireless sensor networks and some of them were described here to relate the proposed algorithm. GA was proposed to improve the network lifetime (Kumar et al 2016), here GA based algorithm is applied to route the data optimally between $\mathrm{CH}$ and BS. But the cluster is same for all round of transmission. Further, bat optimization algorithm was proposed on the basis of local centroid plan to select the cluster heads for choosing $\mathrm{CH}$ selection in WSN (Ahmad et al., 2019). Here, the cluster heads are chosen randomly. PSO algorithm in WSN had been introduced to avoid data redundancies in the base station (Bayrakli et al., 2012). 
ACO was primarily extensively used for cluster design and selection of $\mathrm{CH}$ (Cao et al., 2014) and applied for data aggregation in (Jie et al., 2011). Several bioinspired techniques been implemented by the researchers includes Firefly (Cao et al., 2014), Gravitational search, Local Centroid Bat, Big Bang Crunch (Mosavvar et al., 2019), Flower Pollination (suresh et al., 2020), Cuckoo Search (CS), Ant Colony optimization etc to decide the cluster formation and cluster count. The CS optimization algorithm (Nasir et al., 2017) is applicable for problems with multimodal nature and mainly it is applied to solve localization issues in WSN. Cooperative Multi Input Multi Output (CMIMO) is mostly geared on the selection of nodes as candidates for CNs (Mosavvar et al., 2019) and channel state estimation models however the cooperative routing mostly suffers with channel issues and cooperative nodes selection issues. Modified Queen Honey Bee Migration (mQHBM) was developed (Sharawi et al., 2014) to design an efficient mobile routing in WSN. Here, binary testing injection is used to select the cooperative nodes.

Proposed Mechanism: HBACA: An energy efficient protocol HBACA is proposed to improve the network lifetime of wireless sensor network. An example scenario of the proposed HBACA protocol is given in figure 1. The proposed algorithm follows three phases for such as (i) cluster formation (ii) hauler node selection (iii) Transmission process.

Figure 1: Example Scenario of HBACA

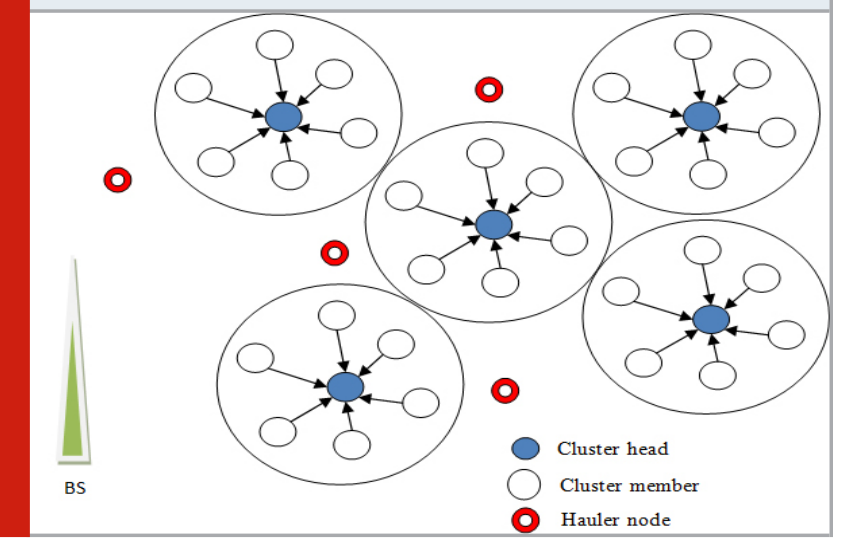

(i) Cluster Formation: The network is divided in to $n$ number of clusters to balance the energy level between the nodes. Genetic Algorithm (GA) is used to create clusters by evaluating the node fitness function includes selection, crossover and mutation. Here in the proposed method the node fitness is calculated using energy and distance parameter of the nodes. The node fitness function (FFNode) is measured using equation 1,

$$
F F_{\text {Node }}=\left[\left(0.4 * F_{1}\right)+\left(0.45 * F_{2}\right)\right]
$$

Based on the behavior of real ants and their search towards the food from their nest the clusters are formed. The cluster members form the cluster by measuring the distance that a particular node can reach to its neighbor node by using ACO method. To maintain the link quality the effective distance is measured between the nodes so that the cluster reformation can be reduced often. The cluster head is chosen by calculating the energy among all the cluster members. Here two kind of energy threshold is set to select the cluster head. Flexible energy threshold and rigid energy threshold are the two types of energy threshold set for the cluster head selection. For each request of base station the data transmission process is carried out for each number of requests. Even after transmission of certain number of rounds the cluster head energy remains under the flexible threshold value $(x<7)$ then there is no need to reform the clusters. If the cluster head falls under the rigid threshold value $(x<3)$ then the cluster reformation should be carried out immediately to avoid the network collapse.

(ii) Hauler node selection: To determine the best hauler node the node cost function is measured. Cuckoo search algorithm is applied to find the hauler node. By calculating the node cost function from the cluster head and the base station the hauler cooperative node can be identified. The node cost is effectively measured through the node cooperativeness with the BS. By considering the received signal strength between the cluster head and the energy level of the respective hauler node; the node cost function is calculated. Therefore the node cost function (CNode) is measured using equation 2.

$$
C_{\text {Node }}=\beta \square\left(R S_{k}\right)+\gamma \square\left(E R_{k}\right)
$$

Here RSk represents the received signal strength of kth cluster's cluster head and ERk referes to the residual energy of the cluster head node.

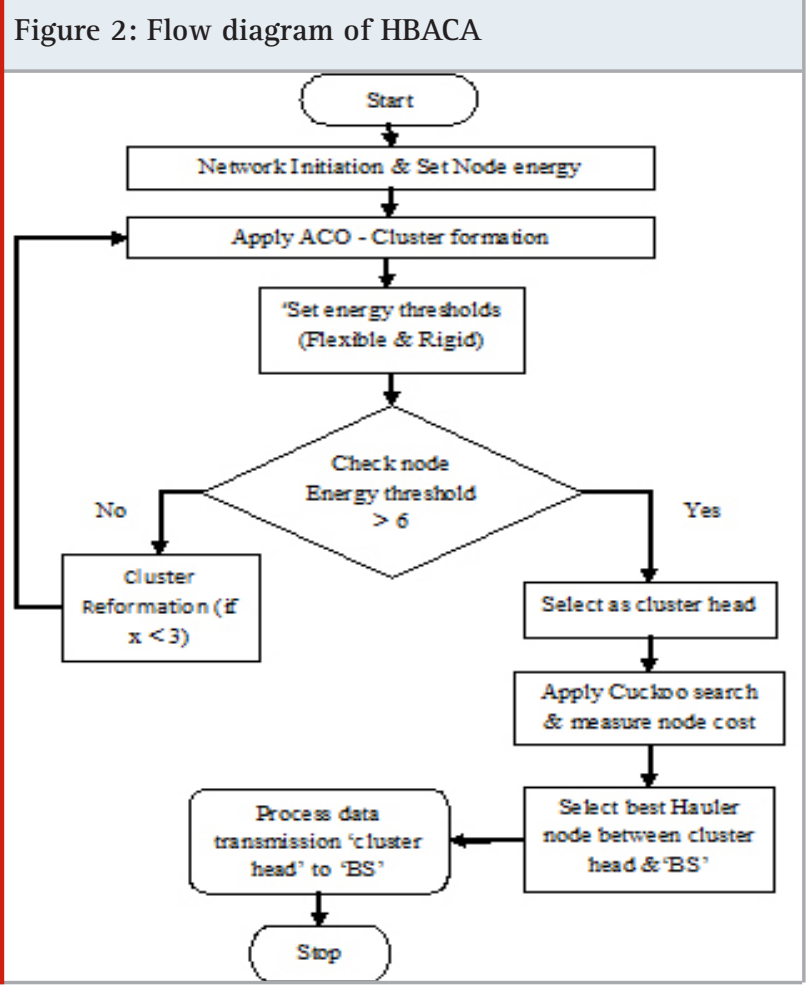


(iii) Transmission Process: Once the cluster formation is done through ACO algorithm and the respective cluster head is elected from all the clusters the sensed data from the cluster members is passed to the cluster head. Then the base station request data from the respective cluster head ' $k$ ' then the cluster head selects the best hauler node by applying cuckoo search algorithm. The data transmission is done from the cluster head to the BS through the hauler node. Thereby for each set of transmission the network follows the same procedure. Figure 2 describes the flow diagram of HBACA protocol.

\section{RESULTS AND DISCUSSION}

The simulator tool used here to simulate the proposed and existing schemes is Network Simulator 2.35. It is a discrete event simulator particularly used for simulating the network communication. By using this tool the validations of protocol proves to be more computational efficient. Scalability factor is more reliable since using the front end process with Object oriented Tool Command Language (OTCL). The network area considered for simulation process is $900 \mathrm{mX} 900 \mathrm{~m}$ dimensions and deployed with of node density 100. The metrics such as Delivery rates of packets (DPR), Average delay and Energy consumption are considered here to evaluate the effectiveness of the proposed protocol HABAC over the conventional scheme mQHBM protocol. Delivery Rate of Packets: The packets that are successfully transmitted over the channel to the base station with respect to the time is said to be delivery rates of packets. It is measured by taking the difference between the total sent packets and total received packets and it is given in equation 3, here the variable $n$ denoted for node count,

$$
D_{P R}=\frac{\sum_{0}^{n} P k t R c v d(n)-\sum_{0}^{n} P k t \operatorname{Sent}(n)}{\text { Time }}
$$

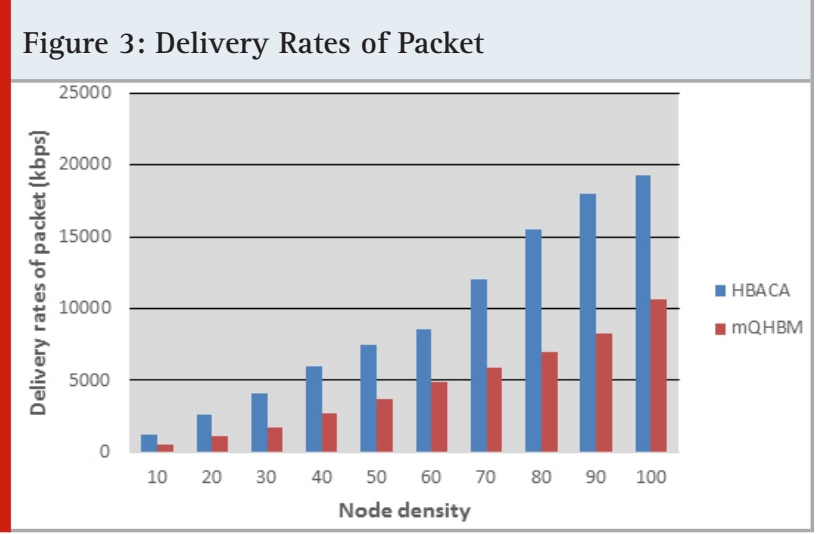

Figure 3 shows the delivery rates of packets for both the proposed and conventional scheme. The proposed scheme HBACA protocol owns good delivery rates of packets when compared with the traditional mQHBM. Average Delay: Average delay can be defined by taking the time variations between the packets that received at present to the packets that received in previous. Equation 4 is used to measure the delay variations.

$$
\text { Average Delay }=\frac{\sum_{0}^{n} P k t \text { RcvdTime }-P k t \text { Sent Time }}{n}
$$

Figure 4 shows the graphical representation of average delay values for the proposed scheme HBACA and the conventional $\mathrm{mQHBM}$. The proposed protocol HBACA attains low delay values for sending and receiving the packets over the channel compared to the conventional scheme.

Energy Consumption: The amount of energy level that is consumed for the processing of data which includes sensing, processing and transmitting is said to be energy consumption. The energy consumption value is carried out for each round of transmission process.

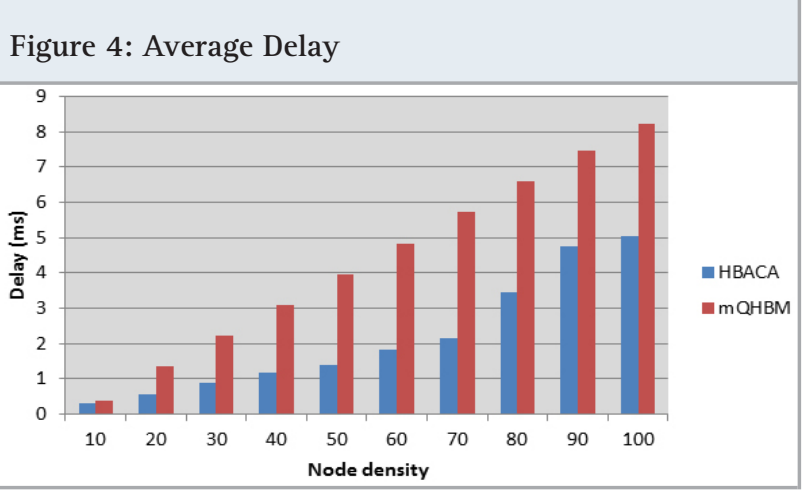

Figure 5: Energy Consumption

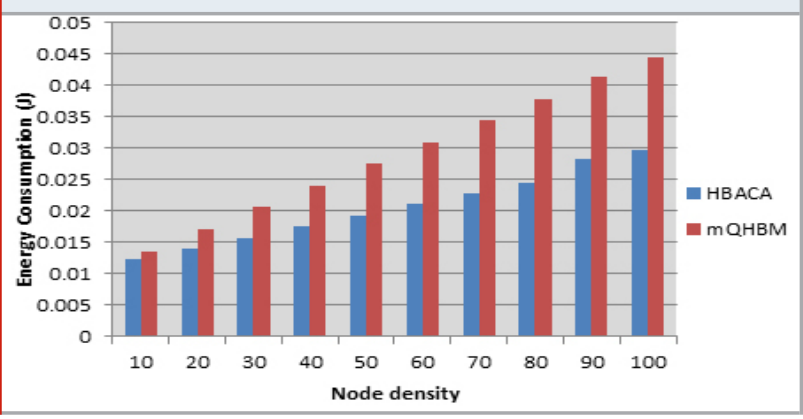

The figure 5 shows the energy consumption value for both the proposed protocol HBACA and existing scheme $\mathrm{mQHBM}$. It is clearly proved that the proposed HBACA scheme consumes low energy for overall process in order to improve the network lifetime when compared with the existing scheme.

\section{CONCLUSION}

An energy efficient protocol HBACA is proposed to balance the energy consumption between the nodes. ACO algorithm is applied for cluster formation. After cluster formation cluster head is selected based upon node energy value. To avoid the frequent cluster reformation flexible and rigid thresholds are set for processing 
successive rounds of transmission with the same cluster head. Node cost is measured by using cuckoo search algorithm and the hauler nodes are selected between cluster head and base station. Thereby efficient routes with reliable nodes are selected for data transmission. Simulation results are shown and the efficiency is proved for the proposed method.

\section{REFERENCES}

Ahmad, T., Haque, M. and Khan, A.M., 2019. An energy-efficient cluster head selection using artificial bees colony optimization for wireless sensor networks. In Advances in nature-inspired computing and applications (pp. 189-203). Springer, Cham.

Bayraklı, S. and Erdogan, S.Z., 2012. Genetic algorithm based energy efficient clusters (gabeec) in wireless sensor networks. Procedia Computer Science, 10, pp.247-254.

Bhatia, T., Kansal, S., Goel, S. and Verma, A.K., 2016. A genetic algorithm based distance-aware routing protocol for wireless sensor networks. Computers \&t Electrical Engineering, 56, pp.441-455.

Cao, Y., Cui, Z., Li, F., Dai, C. and Chen, W., 2014. Improved low energy adaptive clustering hierarchy protocol based on local centroid bat algorithm. Sensor Letters, 12(9), pp.1372-1377.

Das, S., Barani, S., Wagh, S. and Sonavane, S.S., 2017. Optimal clustering and routing for wireless sensor network based on cuckoo search. Int. J. Adv. Smart Sens. Netw. Syst.(IJASSN), 7(2/3), pp.1-13.

Hao, W.Z., Horng, G.J. and Jong, G.J., 2020. A New Bio-Inspired for Cooperative Data Transmission of IoT. IEEE Access, 8, pp.161884-161893.

Izadi, D., Abawajy, J.H., Ghanavati, S. and Herawan, T., 2015. A data fusion method in wireless sensor networks. Sensors, 15(2), pp.2964-2979.

Jie, D., Liu, D.P. and Wu, H.R., 2011. Energy efficiency of virtual MIMO transmission schemes for clusterbased wireless sensor networks. The Journal of china universities of posts and telecommunications, 18(4), pp.31-38.

Kumar, A.S. and Logashanmugam, E., 2016. Secure Acknowledgement based Misbehavior Detection in WSN (S-ACK). Indian Journal of Science and Technology, 9(40), p.96063.

Kumar, A.S. and Logashanmugam, E., 2017. SECURED OPTIMAL ROUTING BASED ON TRUST AND ENERGY MODEL IN WIRELESS SENSOR NETWORKS. IIOAB JOURNAL, 8(3), pp.13-18.

Kumar, A.S., Velmurugan, S. and Logashanmugam, E., 2015. A secure distributed data discovery and dissemination in wireless sensor networks. International Journal of Engineering \& Science Research, 5(7), pp.708713.

Lv, L., 2017. An Improved Ant Colony Algorithm in
Wireless Sensor Network Routing. International Journal of Online Engineering, 13(5).

Mochalov, V., 2015, July. Multi-agent bio-inspired algorithms for wireless sensor network design. In 2015 17th International Conference on Advanced Communication Technology (ICACT) (pp. 33-42). IEEE.

Mosavvar, I. and Ghaffari, A., 2019. Data aggregation in wireless sensor networks using firefly algorithm. Wireless Personal Communications, 104(1), pp.307324.

Nasir, H.J.A., Ku-Mahamud, K.R. and Kamioka, E., 2017. Ant Colony Optimization approaches in wireless sensor network: performance evaluation. Journal of Computer Science, 13(6), pp.153-164.

Ranjeeth, S. and Latchoumi, T.P., 2020. Predicting Kids Malnutrition Using Multilayer Perceptron with Stochastic Gradient Descent. Rev. d'Intelligence Artif., 34(5), pp.631-636.

Rostami, A. and Mottar, M.H., 2014. Wireless sensor network clustering using particles swarm optimization for reducing energy consumption. International Journal of Managing Information Technology, 6(4), p.1.

Senthilkumar, A., Lekashri, S. and Abhay Chaturvedi, D.R.M., 2021. DATA TRAFFIC TRUST MODEL FOR CLUSTERED WIRELESS SENSOR NETWORK. INFORMATION TECHNOLOGY IN INDUSTRY, 9(1), pp.1225-1229.

Senthilkumar, A., Velmurugan, S. and Logashanmugham, E., 2013. Integrating of WLAN/UMTS Network in HotSpot Locations Using OPNET. International Journal of Engineering Research \& Technology (IJERT), 2(11).

Sharawi, M., Emary, E., Saroit, I.A. and El-Mahdy, H., 2014. Flower pollination optimization algorithm for wireless sensor network lifetime global optimization. International Journal of Soft Computing and Engineering, 4(3), pp.54-59.

Suresh, G. and Kumar, A.S., 2020. Secure Transmission Using Bivariate Principle System for WSN. Helix, 10(03), pp.47-51.

Thirunavukkarasu, V., Kumar, A.S., Josephine, D.J. and Arasu, T.P., 2020, July. Selection of Optimistic Nodes for Reputation Based Routing in Wireless Networks. In 2020 7th International Conference on Smart Structures and Systems (ICSSS) (pp. 1-5). IEEE.

Yookesh, T.L., Boobalan, E.D. and Latchoumi, T.P., 2020, March. Variational Iteration Method to Deal with Time Delay Differential Equations under Uncertainty Conditions. In 2020 International Conference on Emerging Smart Computing and Informatics (ESCI) (pp. 252-256). IEEE.

Yuan, X., Elhoseny, M., El-Minir, H.K. and Riad, A.M., 2017. A genetic algorithm-based, dynamic clustering method towards improved WSN longevity. Journal of Network and Systems Management, 25(1), pp.21-46. 